CARDIOVASCULAR MEDICINE

\title{
Physiological upper limits of ventricular cavity size in highly trained adolescent athletes
}

\author{
J Makan, S Sharma, S Firoozi, G Whyte, P G Jackson, W J McKenna
}

Heart 2005;91:495-499. doi: 10.1136/hrt.2004.035121

See end of article for authors' affiliations ......................

Correspondence to: Dr Jayesh Makan, Department of Cardiology, University Hospital Lewisham, Lewisham High Street, London SE13 6LH, UK; jayeshmakan@ btinternet.com

Accepted 10 June 2004

\begin{abstract}
Objectives: To define physiological upper limits of left ventricular (LV) cavity size in trained adolescent athletes.

Design: Cross sectional echocardiographic study.

Setting: British national sports training grounds and Olympic Medical Institute.

Subjects: 900 elite adolescent athletes (77\% boys) aged 15.7 (1.2) years participating in ball, racket, and endurance sports and 250 healthy controls matched for age, sex, and size.

Main outcome measures: LV end diastolic cavity size.

Results: Compared with controls, athletes had a larger LV cavity $(50.8$ (3.7) v 47.9 (3.5) mm), a difference of $6 \%$. The LV cavity was $>54 \mathrm{~mm}$ in $18 \%$ athletes, whereas none of the controls had an LV cavity $>54 \mathrm{~mm}$. The LV cavity exceeded predicted sizes in $117(13 \%)$ athletes. Among the athletes with LV dilatation, $78 \%$ were boys, LV size ranged from $52-60 \mathrm{~mm}$, and left atrial diameter and LV wall thickness were enlarged. Systolic and diastolic function were normal. None of the athletes in the study had an LV cavity size $>60 \mathrm{~mm}$. LV cavity size correlated with age, sex, heart rate, and body surface area.

Conclusion: Highly trained junior athletes usually have only modest increases in LV cavity size. A proportion of trained adolescent athletes have LV cavity size exceeding predicted values but, in absolute terms, LV cavity rarely exceeds $60 \mathrm{~mm}$ as in patients with dilated cardiomyopathy. In highly trained adolescent athletes with an LV cavity size $>60 \mathrm{~mm}$ and any impairment of systolic or diastolic function, the diagnosis of dilated cardiomyopathy should be considered.
\end{abstract}

R egular intensive physical exercise is associated with an increase in left ventricular (LV) wall thickness and cavity size. ${ }^{1-6}$ In a proportion of athletes the magnitude of LV cavity enlargement may be substantial, creating uncertainty regarding the differential diagnosis of athlete's heart (physiological cardiac enlargement) and dilated cardiomyopathy (DCM), ${ }^{7}$ especially when the indices of systolic function at rest are at the lower limits of normally accepted values. The differentiation between athlete's heart and DCM is important when one considers that DCM is a recognised cause of sudden death in the young athletic population. ${ }^{8}$

The definition of the upper limits of physiological cavity enlargement in athlete's heart is based on echocardiographic studies of adult athletes. ${ }^{1-6} 9$ Reference values for upper limits of LV cavity dimensions derived from studies of adult athletes cannot be extrapolated to younger athletes for the purpose of differentiating physiological ventricular cavity dilatation from DCM, as junior athletes are physically less mature and generally exposed to a shorter period of training. ${ }^{10}$ This study was undertaken to define the physiological limits of LV cavity size in elite adolescent athletes to facilitate the differentiation between athlete's heart and pathological LV cavity dilatation.

\section{METHODS \\ Athletes}

Between August 1996 and November 2003, 900 post-pubertal adolescent elite athletes aged 15.7 (1.2) years (range 14-18 years) underwent two dimensional echocardiography during the competitive season. Six hundred and sixty four (74\%) were boys; 882 (98\%) were white. Body surface area (BSA) among the athletes was $1.74(0.16) \mathrm{m}^{2}$ (range 1.09-2.24 $\mathrm{m}^{2}$ ).

Written consent for cardiovascular evaluation was obtained from participants aged $\geqslant 16$ years and from a parent or guardian for those $<16$ years old.
The study group was predominantly made up of 10 sporting disciplines: boxing, cycling, hockey, karate, rowing, rugby, soccer, swimming, tennis, and triathlon. Soccer and tennis were the most commonly studied sports and between them accounted for $414(46 \%)$ athletes (table 1). Tennis players were recruited from the British Lawn Tennis Association, soccer players from youth teams at clubs in the British Premier Soccer League, boxers from prominent amateur boxing association clubs, rugby players from British Rugby League clubs, cyclists from large county cycling squads, triathletes from top 10 finishers at the national UK championships, and rowers, swimmers, and hockey and karate players from British junior national teams. An additional $18(2 \%)$ had participated at the national level in squash, fencing, and track and field events.

All athletes had competed at the regional level for a mean (SD) of 4.3 (1.5) years (range 1-10 years) and 50\% were competing at the national level at time of this study. The

Table 1 Characteristics of 900 junior elite athletes undergoing echocardiography

\begin{tabular}{llrrrl}
\hline Sport & Total $(\mathbf{n}=900)$ & Boys & Girls & Age (years) & BSA $\left(\mathbf{m}^{2}\right)$ \\
\hline Tennis & $225(25 \%)$ & 110 & 115 & $15.4(1.1)$ & $1.70(1.1)$ \\
Soccer & $189(21 \%)$ & 189 & 0 & $16.0(1.2)$ & $1.76(1.2)$ \\
Cycling & $108(12 \%)$ & 87 & 23 & $15.6(1.2)$ & $1.73(0.4)$ \\
Rugby & $90(10 \%)$ & 90 & 0 & $16.4(1.1)$ & $1.78(1.3)$ \\
Swimming & $90(10 \%)$ & 45 & 45 & $15.5(0.9)$ & $1.71(0.1)$ \\
Karate & $54(6 \%)$ & 35 & 19 & $15.7(1.0)$ & $1.73(0.3)$ \\
Rowing & $45(5 \%)$ & 45 & 0 & $16.6(0.6)$ & $1.88(0.2)$ \\
Hockey & $36(4 \%)$ & 14 & 22 & $15.7(1.2)$ & $1.73(0.4)$ \\
Triathlon & $27(3 \%)$ & 17 & 10 & $16.6(1.1)$ & $1.70(1.3)$ \\
Boxing & $18(2 \%)$ & 18 & 0 & $16.5(1.3)$ & $1.76(1.3)$ \\
Others & $18(2 \%)$ & 14 & 4 & $15.8(0.7)$ & $1.73(0.4)$ \\
\hline
\end{tabular}

Values are mean (SD) or number (\%).

BSA, body surface area. 
Table 2 Predicted upper levels of left ventricular (LV) cavity size in healthy adolescents aged 14-18 years based on 250 controls

\begin{tabular}{llllll}
\hline & \multicolumn{2}{l}{ Boys } & & \multicolumn{2}{l}{ Girls } \\
\cline { 6 - 6 } \cline { 5 - 5 } Age (years) & LVEDD (mm) & Predicted upper limit (2SD) & & \\
& & & & \\
& & & & & \\
14 & $46.1(4.0)$ & 54.1 & $44.7(2.9)$ & 50.4 \\
15 & $49.8(2.2)$ & 54.2 & $46.3(3.2)$ & 52.8 \\
16 & $50.4(2.6)$ & 55.7 & $46.5(2.9)$ & 52.3 \\
17 & $50.3(2.2)$ & 54.7 & $46.5(2.3)$ & 51.0 \\
18 & $49.0(3.9)$ & 56.7 & $43.3(3.1)$ & 50.4 \\
\hline
\end{tabular}

number of hours of intensive training averaged 10.6 (3.5) hours/week (range 6-30 hours). No athlete had symptoms of underlying cardiovascular disease or a family history of premature (aged $<50$ years) sudden death from heart disease, and none had a blood pressure exceeding $130 \mathrm{~mm} \mathrm{Hg}$ systolic or $85 \mathrm{~mm} \mathrm{Hg}$ diastolic.

\section{Controls}

The control population comprised 250 healthy adolescent volunteers who were students at two large secondary education boarding schools. All selected participants led a relatively sedentary lifestyle, defined as $<2$ hours of organised physical activity a week. Controls were well matched to the trained athletes with regard to age, sex, and BSA (15.5 (1.2) years (range 14-18 years), 71\% boys, $1.71(0.4) \mathrm{m}^{2}$ (range $1.17-2.24 \mathrm{~m}^{2}$ ), respectively, not significant).

\section{Echocardiography}

Two dimensional echocardiograms were recorded with a computed sonograph (128XP/10c, Acuson, San Jose, California, USA) with a $3 \mathrm{MHz}$ transducer. Images of the heart were obtained in the standard parasternal long axis, short axis, and apical four chamber planes. ${ }^{11}$ The LV wall thickness was measured from the short axis views of the anterior septum and LV posterior wall in end diastole at the mitral valve and papillary muscle levels. LV end diastolic and end systolic diameters were measured from short axis views at the tips of the mitral valve. All measurements were made on line by two authors (SS and SF) with the aid of a calibration scale provided by the machine. M mode echocardiograms were derived from two dimensional images in the parasternal long axis to assist in the measurement of the left atrial and aortic root diameters according to standards set by the American Society of Echocardiography. ${ }^{12}$ The LV shortening fraction was calculated as an index of systolic function. Pulsed Doppler images were recorded at the level of the mitral valve annulus as described previously to provide an index of diastolic function. ${ }^{13}$ Continuous wave Doppler images were recorded across the LV inflow and outflow tract. ${ }^{14}$ LV mass was calculated from the LV cavity size and wall thickness in end diastole by the formula by Devereux. ${ }^{15}$

Predicted upper limits for normal LV cavity size were derived from the control group. The predicted upper limits were defined as two standard deviations from the mean (table 2).

In accordance with usual clinical practice we relied on absolute LV end diastolic cavity sizes; however, for selective comparisons of sex, age, and sporting disciplines, measurements were corrected for differences in BSA.

\section{Statistical analysis}

Statistical analyses were performed by unpaired Student's $t$ test, analysis of variance, $\chi^{2}$ test, correlation coefficient, and linear regression where appropriate. Significance was defined by $\mathrm{p}<0.05$.

\section{RESULTS}

\section{LV cavity size and LV function in athletes versus} controls

Athletes had a significantly larger LV cavity (fig 1), LV wall thickness, and left atrial diameter than the control group (table 3 ). The percentage difference in mean absolute LV

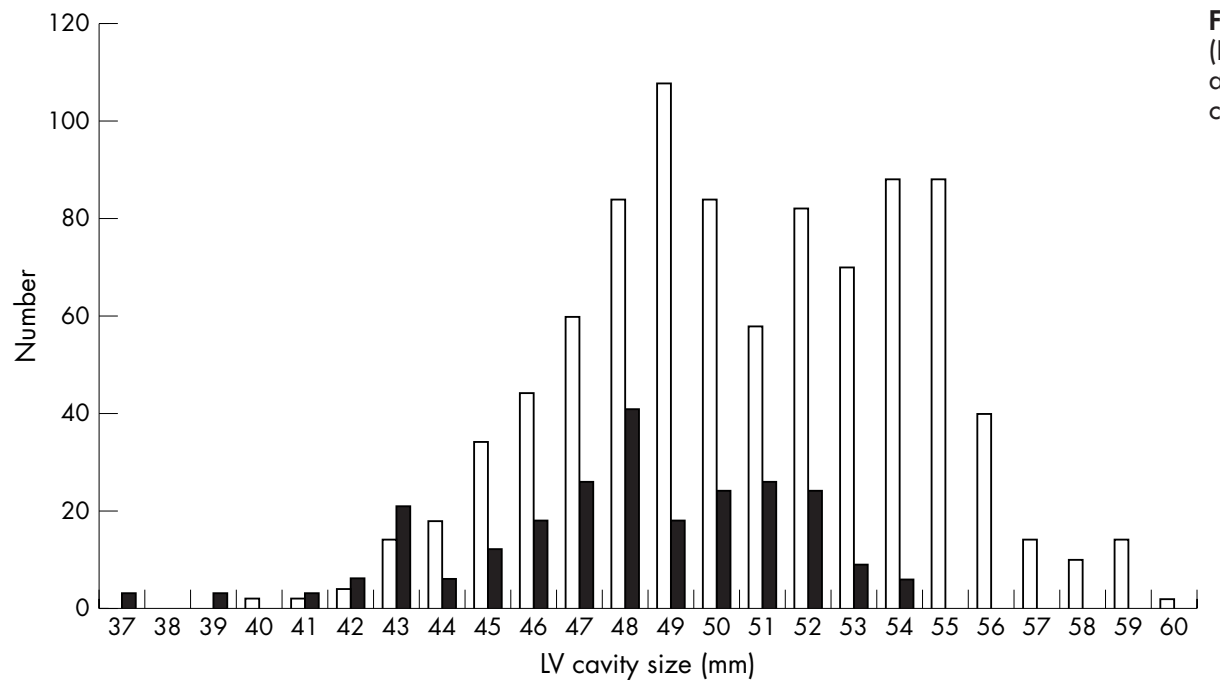

Figure 1 Distribution of left ventricular (LV) cavity size in 900 junior elite athletes (open bars) and 250 healthy controls (solid bars). 
Table 3 Echocardiographic dimensions in junior elite athletes and controls

\begin{tabular}{llll}
\hline & Athletes & Controls & p Value \\
\hline Age (years) & $15.7(1.2)$ & $15.5(1.2)$ & NS \\
$\quad$ Range & $(14-18)$ & $(14-18)$ & \\
BSA (m $\left.{ }^{2}\right)$ & $1.74(0.16)$ & $1.71(0.4)$ & NS \\
$\quad$ Range & $(1.09-2.23)$ & $(1.17-2.24)$ & \\
Heart rate (beats/min) & $52.7(5.7)$ & $70.7(10.6)$ & $<0.05$ \\
MLVWT (mm) & $9.6(1.3)$ & $8.5(1.3)$ & $<0.05$ \\
Range & $(6-14)$ & $(6-11)$ & \\
LVEDD (mm) & $50.8(3.7)$ & $47.9(3.5)$ & $<0.05$ \\
Range & $(40-60)$ & $(37-54)$ & \\
LVEDDI (mm/m $\left.{ }^{2}\right)$ & $29.3(2.3)$ & $28.3(2.6)$ & $<0.05$ \\
Left atrial diameter (mm) & $32.7(4.8)$ & $30.9(5.0)$ & $<0.05$ \\
$\quad$ Range & $(19-45)$ & $(20-40)$ & \\
Aortic root diameter (mm) & $28.7(3.5)$ & $27.1(3.5)$ & NS \\
$\quad$ Range & $(17-40)$ & $(19-33)$ & \\
LVSF (\%) & $33.1(3.5)$ & $31.9(3.9)$ & NS \\
Range & $(31-40)$ & $(30-39)$ & \\
E wave (m/s) & $0.87(0.16)$ & $0.88(0.2)$ & NS \\
$\quad$ Range & $(0.5-1.7)$ & $(0.6-1.8)$ & \\
A wave (m/s) & $0.41(0.1)$ & $0.45(0.16)$ & $<0.05$ \\
Range & $(0.2-0.9)$ & $(0.2-0.8)$ & \\
E:A ratio & $2.25(0.7)$ & $2.14(0.16)$ & NS \\
Range & $(1.0-5.5)$ & $(1.2-4.5)$ & \\
\hline
\end{tabular}

Values are mean (SD).

LVEDD, left ventricular end diastolic diameter; LVEDDI, left ventricular cavity size adjusted for BSA; LVSF, left ventricular shortening fraction; MLVWT, maximum left ventricular wall thickness; NS, not significant.

cavity size between athletes and non-athletes was 6\%. None of the 250 participants in the control group had an LV cavity > $54 \mathrm{~mm}$. In contrast, LV cavity exceeded $54 \mathrm{~mm}$ in $162(18 \%)$ athletes. Indices of systolic and diastolic function were normal in athletes and non-athletes. There were no significant differences in parameters of systolic or diastolic function between athletes and non-athletes (table 3).

\section{LV cavity size in athletes}

The LV cavity size in athletes ranged from $40-60 \mathrm{~mm}$ (fig 1). Boys had a larger absolute LV cavity size than girls (51.6 (3.3) v 47.7 (3.3) mm, p < 0.05). In terms of absolute values, none of the female athletes had an absolute LV cavity $>55 \mathrm{~mm}$. In contrast, LV cavity exceeded $55 \mathrm{~mm}$ in 73 (11\%) male athletes (fig 2). There was no significant difference in mean LV cavity size between athletes of different ages. Male rowers had a greater absolute LV cavity size than other athletes, but this difference was abolished by correction for BSA.
Athletes with LV cavity size exceeding predicted limits The vast majority of athletes had an absolute LV cavity size falling within normal values derived from controls (table 4). However, 117 (13\%) athletes had a LV cavity exceeding the predicted upper limits of normal. The LV cavity in these 117 athletes ranged from $52-60 \mathrm{~mm}$.

\section{Demographics}

Of these 117 athletes with an enlarged LV cavity, 91 (78\%) were boys. Athletes in this group were slightly older (15.7 (1.2) $v 15.6(1.3)$ years, $\mathrm{p}<0.05)$ and had a greater BSA $\left(1.84(0.18) v 1.73(0.16) \mathrm{m}^{2}, \mathrm{p}<0.05\right)$ than athletes with a normal LV cavity. After correction for BSA, the mean LV cavity in the 117 athletes remained greater than that in athletes with a normal LV cavity (30.7 (2.5) v 29.1 (2.2) mm/ $\left.\mathrm{m}^{2}, \mathrm{p}<0.05\right)$. Athletes participating in rowing, swimming, soccer, tennis, triathlon, athletics, badminton, and speed skating were represented in this group. A significant proportion of rowers and cyclists ( $43 \%$ and $41 \%$, respectively) had an enlarged LV cavity.

\section{Echocardiographic parameters}

Athletes with an enlarged LV cavity also had a larger mean LV wall thickness (10.1 (1.3) v 9.5 (1.3) mm, p < 0.05) and mean left atrial diameter (34.8 (4.0) v 32.4 (4.8) mm, $\mathrm{p}<0.05)$ than did athletes with a normal sized LV cavity. The mean shortening fraction in athletes with an enlarged LV cavity was 37 (4)\% (range 32-43\%). Athletes with an enlarged LV cavity had normal indices of systolic and diastolic function that did not differ from those of athletes with a normal LV cavity.

\section{DISCUSSION}

Echocardiographic studies of adult athletes have defined upper limits of physiological LV cavity enlargement; however, data regarding LV cavity size in younger athletes are limited. Apart from being of physiological interest, information regarding the upper limits of LV cavity size in elite adolescent athletes may be useful in enabling the differentiation between physiological LV cavity enlargement (part of athlete's heart) and DCM. The differentiation is particularly important in instances where indices of systolic function are at the lower limit of the normally acceptable range in an athlete in question or when an athlete is experiencing symptoms that may be consistent with an underlying cardiac problem. Our study of 900 adolescents aimed at defining upper limits of physiological LV cavity enlargement in highly trained adolescent athletes aged 14-18 years.

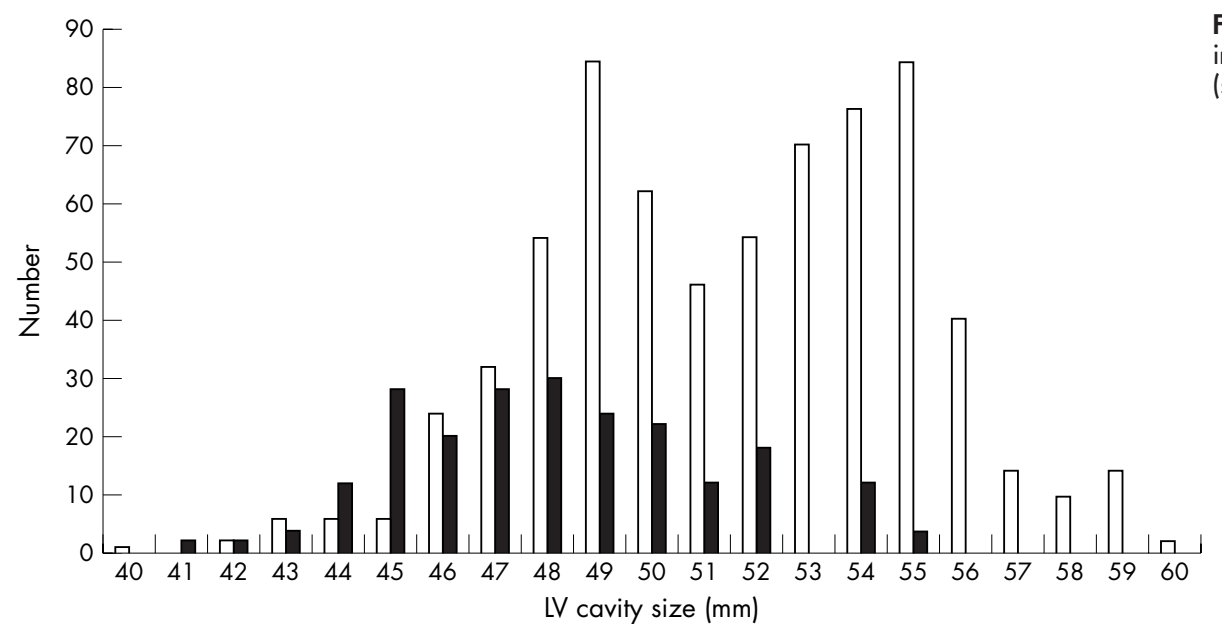

Figure 2 Distribution of LV cavity size in junior male (open bars) and female (solid bars) athletes.

$\mathrm{LV}$ cavity size $(\mathrm{mm})$ 
Table 4 Comparison of demographic and echocardiographic variables between athletes with enlarged LV cavity and athletes with LV cavity size within predicted limits

\begin{tabular}{|c|c|c|c|}
\hline & $\begin{array}{l}\text { LV cavity size within } \\
\text { normal limits }\end{array}$ & $\begin{array}{l}\text { LV cavity size exceeding } \\
\text { predicted upper limits }\end{array}$ & p Value \\
\hline Number & 783 & 117 & \\
\hline Age (years) & $15.6(1.3)$ & $15.7(1.2)$ & $<0.05$ \\
\hline Height & $171.0(8.5)$ & $175.4(9.3)$ & $<0.05$ \\
\hline Weight (kg) & $62.5(9.2)$ & $68.8(10.6)$ & $<0.05$ \\
\hline BSA $\left(\mathrm{m}^{2}\right)$ & $1.73(0.16)$ & $1.84(0.18)$ & $<0.05$ \\
\hline Heart rate (beats $/ \mathrm{min}$ ) & $53.7(5.2)$ & $45.7(3.8)$ & $<0.05$ \\
\hline Training intensity (hours/week) & $4.32(1.45)$ & $4.44(1.85)$ & \\
\hline Left atrial diameter $(\mathrm{mm})$ & $32.4(4.8)$ & $34.8(4.0)$ & $<0.05$ \\
\hline LVEDD $(\mathrm{mm})$ & $50.0(3.3)$ & $55.8(2.0)$ & $<0.05$ \\
\hline MLVWT (mm) & $9.5(1.3)$ & $10.1(1.3)$ & $<0.05$ \\
\hline Aortic root diameter $(\mathrm{mm})$ & $28.6(3.5)$ & $30.0(3.4)$ & $<0.05$ \\
\hline LV mass (g) & $190.3(60.1)$ & $262.9(66.1)$ & $<0.05$ \\
\hline$E$ wave $(\mathrm{m} / \mathrm{s})$ & $0.86(0.15)$ & $0.91(0.17)$ & $<0.05$ \\
\hline A wave $(\mathrm{m} / \mathrm{s})$ & $0.41(0.11)$ & $0.43(0.11)$ & NS \\
\hline E:A ratio & $2.25(0.72)$ & $2.19(0.63)$ & NS \\
\hline
\end{tabular}

\section{Athletes with enlarged LV cavity}

Our athletes had modest increases in LV cavity size compared with non-athletes. Although the majority of athletes in the study had a normal LV cavity (usually $\leqslant 54 \mathrm{~mm}$ ), an important minority 117 (13\%) had an LV cavity exceeding predicted limits. The LV cavity did not exceed $60 \mathrm{~mm}$ in any athlete irrespective of age, size, sex, sporting discipline, and duration of participation in sport or athletic achievement (in terms of representation at the national level). Therefore, extreme forms of LV remodelling resulting in $\mathrm{LV}>60 \mathrm{~mm}$ in junior athletes should raise the possibility of underlying DCM in athletes with diminished indices of systolic function on the resting echocardiogram. Sex differences may prove pertinent in the differentiation between physiological and pathological LV enlargement. In our study most athletes with an enlarged LV cavity were boys. Although 12\% of girls had an enlarged LV cavity, none had an LV > $>55 \mathrm{~mm}$. Consequently, an $\mathrm{LV}>55 \mathrm{~mm}$ in a female junior athlete should raise the suspicion of DCM, particularly if systolic or diastolic parameters are abnormal.

Our 117 athletes with an enlarged LV cavity had several echocardiographic features permitting differentiation from DCM. All 117 athletes had normal indices of systolic and diastolic function. In contrast most young patients with DCM have impaired systolic or diastolic LV dysfunction. Our 117 athletes with increased LV cavity size had increased LV wall thickness and left atrial diameter, which may accompany both physiological and pathological LV enlargement. Normal systolic function in our athletes suggested that the increased LV wall thickness indicated physiological LV hypertrophy rather than the compensatory hypertrophy seen in DCM. Normal mitral inflow velocities indicated that increased left atrial diameter was also part of general physiological cardiac enlargement rather than diastolic dysfunction secondary to DCM.

\section{Determinants of LV cavity size in athletes}

The study relied on absolute cardiac dimensions to place the results in context with usual clinical practice. Our multivariate analysis showed that BSA, sex, and heart rate were independent determinants of LV cavity size. Individual cardiac dimensions in athletes varied within the same sport even after allowing for these determinants indicating that some other factors, possibly genetic, may also be important in determining the cardiac adaptation to exercise. ${ }^{16}{ }^{17}$

The duration of participation in competition and number of hours of training a week did not correlate with cavity dimensions. A possible explanation for this is that neither is a reliable index of intensity of training. A more accurate way may have been to objectively assess each athlete's training schedule. An alternative explanation may be that cavity dimensions are genetically determined and that people with larger cavity size are naturally selected to excel in sport. However, longitudinal studies of athletes have shown that cavity dimensions do increase with training and regress with detraining. ${ }^{18-20}$ Furthermore, studies of pre-pubertal children have not found any difference in cavity dimensions between athletes and controls to suggest natural selection of people with large hearts. ${ }^{21} 22$

\section{Difference in LV cavity size between adult and adolescent athletes}

Adolescent athletes were similar to adult athletes in many respects. For example, LV cavity was largest in boys with large BSAs. As with adult athletes, adolescent athletes participating in endurance events such as rowing and cycling usually had LV cavity sizes exceeding predicted limits. However, the difference in LV size between athletes and nonathletes was considerably smaller among adolescent athletes than observed among adult athletes. ${ }^{1}$ Also, whereas $45 \%$ adult athletes have an LV cavity $\geqslant 55 \mathrm{~mm}$ and $14 \%$ have an LV cavity $\geqslant 60 \mathrm{~mm},{ }^{7}$ only $18 \%$ of adolescent athletes had an LV cavity $\geqslant 55 \mathrm{~mm}$ and only one $(0.1 \%)$ athlete had an LV cavity $\geqslant 60 \mathrm{~mm}$. The largest LV cavities in adult male and female athletes were $70 \mathrm{~mm}$ and $66 \mathrm{~mm}$, respectively, but none of our male athletes had an LV cavity $>60 \mathrm{~mm}$ and none of the female athletes had an LV cavity $>55 \mathrm{~mm}$. These fundamental differences in LV cavity size between adult and adolescent athletes probably reflect the relative lack of physical maturity and fewer cumulative hours of training among adolescent athletes. These differences underscore the importance of using separate cut off values for adolescent athletes when differentiating between physiological LV cavity enlargement and DCM.

This study was limited by the fact that only athletes participating in popular sports in the UK were evaluated, whereas common US sports such as basketball and American football were not represented. Similarly, athletes performing predominantly isometric training such as power lifters were not studied because of the difficulty obtaining large numbers of junior athletes participating at the national level. Furthermore, almost all (98\%) of our study population was white and therefore the effects of racial variation could not be studied. Despite these limitations, this study provides valuable information regarding the upper limit of LV cavity size in the highly trained junior athlete and will prove clinically 
useful in the differentiation of physiological adaptation from cardiomyopathy in this important group of people.

\section{ACKNOWLEDGEMENTS}

The authors acknowledge the invaluable help of Cardiac Risk in the Young (CRY) in providing ECG and echocardiography equipment for this study. Dr S Sharma and Dr S Firoozi were supported by grants from the British Heart Foundation. Dr J Makan is supported by a grant from CRY.

\section{Authors' affiliations}

J Makan, S Sharma, S Firoozi, G Whyte, P G Jackson, W J McKenna, University Hospital Lewisham, London, UK

\section{REFERENCES}

1 Pluim BM, Zwinderman AH, van der Laarse A, et al. The athlete's heart: a meta-analysis of cardiac structure and function. Circulation 2000;101:336-44.

2 Maron BJ. Structural features of the athlete heart as defined by echocardiography. J Am Coll Cardiol 1986;7:190-203.

3 Rost R. The athlete's heart. Eur Heart J 1982;3(suppl A):193-8.

4 Huston TP, Puffer JC, Rodney WM. The athletic heart syndrome. N Engl J Med 1985;313:24-32

5 Fagard R, Aubert A, Lysens R, et al. Noninvasive assessment of seasonal variation in cardiac structure and function in cyclists. Circulation 1983;67:896-901.

6 Nishimura T, Yamada Y, Kawai C. Echocardiographic evaluation of long-term effects of exercise on left ventricular hypertrophy and function in professional bicyclists. Circulation 1980;61:832-40.

7 Pelliccia A, Culasso F, Di Paolo FM, et al. Physiologic left ventricular cavity dilatation in elite athletes. Ann Intern Med 1999;130:23-31.

8 Maron BJ, Shirani J, Poliac LC, et al. Sudden death in young competitive athletes. Clinical, demographic and pathological profiles. JAMA 1996;276:199-204.
9 Spirito P, Pelliccia A, Proschan MA, et al. Morphology of the "athlete heart" assessed by echocardiography in 947 elite athletes representing 27 sports. Am J Cardiol 1994;74:802-6.

10 Wieling W, Borghols EA, Hollander AP, et al. Echocardiographic dimensions and maximal oxygen uptake in oarsmen during training. Br Heart $J$ $1981 ; 46: 190-5$.

11 Tajik AJ, Seward JB, Hagler DJ, et al. Two dimensional real-time ultrasonic imaging of the heart and great vessels: technique, image orientation, structure, identification and validation. Mayo Clin Proc 1978;53:271-303.

12 Sahn DJ, DeMaria A, Kisslo J, et al. Recommendations regarding quantitation in M-mode echocardiography: results of a survey of echocardiographic measurements. Circulation 1978;58:1072-83.

13 Spirito P, Maron BJ. Doppler echocardiography for assessing left ventricular diastolic function. Ann Intern Med 1988;109:122-6.

14 Maron BJ, Gottdiener JS, Arce J, et al. Dynamic subaortic obstruction in hypertrophic cardiomyopathy: analysis by pulsed Doppler echocardiography. $J$ Am Coll Cardiol 1985;6:1-18.

15 Devereux RB. Detection of left ventricular hypertrophy by M-mode echocardiography: anatomic validation, standardization and comparison to other methods. Hypertension 1987;9:119-26.

16 Adams TD, Yanowitz FG, Fisher AG, et al. Heritability of cardiac size: an echocardiographic and electrocardiographic study of monozygotic and dizygotic twins. Circulation 1985;71:39-44.

17 Klissouras V, Pirnay F, Petit JM. Adaptation to maximal effort: genetics and age. J Appl Physio 1973;35:288-93.

18 Ehsani AA, Hagberg JM, Hickson RC. Rapid changes in left ventricular dimensions and mass in response to physical conditioning and deconditioning. Am J Cardiol 1978;42:52-6.

19 De Maria AN, Neumann A, Lee G, et al. Alterations in ventricular mass and performance induced by exercise training in man evaluated by echocardiography. Circulation 1978;57:237-44.

20 Shapiro LM, Smith RG. Effect of training on left ventricular structure and function: an echocardiographic study. Br Heart J 1983;50:534-9.

21 Allen HD, Goldberg SJ, Sahn DJ, et al. A quantitative echocardiographic study of champion childhood swimmers. Circulation 1977;55:142-4.

22 Rowland TW, Delaney BC, Siconolfi SF. 'Athlete's heart' in prepubertal children. Pediatrics 1987;79:800-4.

\section{IMAGES IN CARDIOLOGY}

\section{Three dimensional transthoracic echocardiography images of tricuspid stenosis}

\begin{abstract}
A 56 year old man was admitted for evaluation of exertional dyspnoea. His medical history was significant for rheumatic mitral and aortic valve disease. A transthoracic three dimensional (3D) echocardiography examination was performed using a Philips Sonos 7500 (Philips Medical Systems, Eindhoven, the Netherlands) and a new $4 \mathrm{MHz}, 4 \mathrm{X}$ matrix transducer capable of providing real time two dimensional (2D) and live 3D transthoracic images. Pyramidal shaped full volume 3D images were acquired with ECG gating by asking the patient to hold his breath for a few seconds. The system permits cropping of $3 \mathrm{D}$ images in three different orthogonal axes (lateral, elevational, and depth). The image was cropped using an elevational cutting plane to obtain the profile of the tricuspid valve area viewed from the apex perspective. Three dimensional images were then digitally stored on optical disk and transferred into a dedicated computer (Tom-TEC, EchoView) for measurements. A valve area of $1.4 \mathrm{~cm}^{2}$ was measured.

Tricuspid stenosis is a rare clinical condition with rheumatic disease accounting for about $90 \%$ of all cases. Two dimensional echocardiography permits definitive diagnosis of tricuspid stenosis showing thickening and shortening of the valve leaflets. Nevertheless, unlike evaluation of mitral stenosis, short axis $2 \mathrm{D}$ imaging of the valve
\end{abstract}

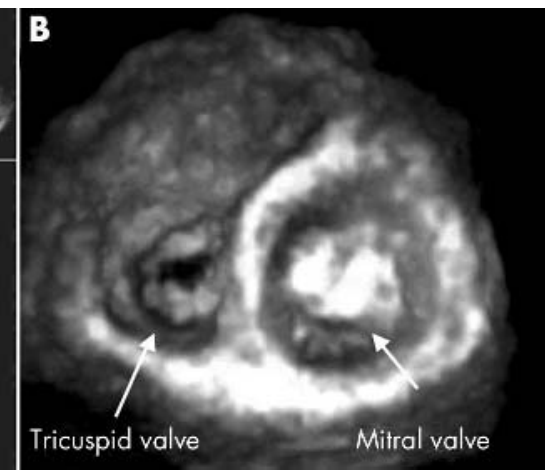

Using four ECG triggered cardiac beats with the patient breath holding, four subvolumes were time aligned to render a pyramidal shaped full volume image (panel A, top right corner). To obtain the tricuspid valve area from the ventricular perspective, the three dimensional full volume was cropped using the elevational plane from the apex.

orifice is rarely feasible. In this patient, an image of tricuspid valve area was easily obtained using 3D transthoracic echocardiography. To our knowledge these images have not been reported previously.

F Faletra

U La Marchesina

R Bragato

F De Chiara francesco.faletra@humanitas.it 